\title{
Advanced 1D Structural Models for Flutter Analysis of Lifting Surfaces
}

\author{
Marco Petrolo* \\ Department of Mechanical and Aerospace Engineering, Politecnico di Torino, Torino, Italy
}

\begin{abstract}
An advanced aeroelastic formulation for flutter analyses is presented in this paper. Refined 1D structural models were coupled with the doublet lattice method, and the g-method was used for flutter analyses. Structural models were developed in the framework of the Carrera Unified Formulation (CUF). Higher-order 1D structural models were obtained by using Taylor-like expansions of the cross-section displacement field of the structure. The order $(\mathrm{N})$ of the expansion was considered as a free parameter since it can be arbitrarily chosen as an input of the analysis. Convergence studies on the order of the structural model can be straightforwardly conducted in order to establish the proper 1D structural model for a given problem. Flutter analyses were conducted on several wing configurations and the results were compared to those from literature. Results show the enhanced capabilities of CUF ID in dealing with the flutter analysis of typical wing structures with high accuracy and low computational costs.
\end{abstract}

Key words: Unified Formulation, Beam, Doublet Lattice Method, G-Method

\section{Introduction}

Flutter is one of the most important aeroelastic phenomena. Flutter can occur to a structure in a flow field, and it consists of undamped vibrations that can lead to catastrophic collapses. This implies that flight vehicles or bridges, for instance, must be clear of flutter. Different analysis tools have been developed over the last decades to predict flutter. A vast range of aerodynamic models have been utilized in aeroelastic problems, from strip theories to Reynolds-averaged NavierStokes (RANS). Excellent reviews about these methodologies are presented in [1] and [2]. One of the first methods for flutter analysis was strip theory [3-4]. From the early 1940s to the 1960 s, strip theory and its variations represented the most important tools for flutter. The doublet lattice method (DLM) emerged in the late 1960s [5]. More recently, an improved version of DLM has been proposed by Rodden [6], and it is this version that is utilized in this work. To date, DLM is one of the most powerful tools for linear flutter analyses in the subsonic regime. Three main features are responsible for DLM's success [1]:

1. It offers good accuracy (unless transonic regimes are considered and/or separation occurs).

2. DLM is cost competitive with respect to simpler methods such as strip theories.

3. Fairly complex geometries can be analyzed.

The structural component of the aeroelastic formulation adopted in this paper was based on 1D higher-order structural models commonly known as beams. Beam theories are extensively used to analyze the structural behavior of slender bodies, such as columns, arches, blades, aircraft wings and bridges. In a beam model, the $3 \mathrm{D}$ problem is reduced to a set of variables that only depends on the beam-axis coordinate. One-dimensional structural elements are simpler and computationally more efficient than 2D (plate/shell) and 3D (solid) elements. This feature makes beam theories still very attractive for the static, dynamic and aeroelastic analysis of structures.

The classical theories are those by Euler-Bernoulli [7-8]

This is an Open Access article distributed under the terms of the Creative Commons Attribution Non-Commercial License (http://creativecommons.org/licenses/by$\mathrm{nc} / 3.0 /$ which permits unrestricted non-commercial use, distribution, and reproduction in any medium, provided the original work is properly cited. 
and Timoshenko [9]. None of these theories can detect non-classical effects such as warping, out- and in-plane deformations, torsion-bending coupling or localized boundary conditions (geometrical or mechanical). These effects are important when, for instance, small slenderness ratios, thin walls and the anisotropy of the materials are considered. An accurate aeroelastic analysis requires the proper detection of non-classical effects.

Many methods have been proposed to overcome the limitations of classical theories and to extend the application of $1 \mathrm{D}$ models to any geometry or boundary conditions [10]. Most recent developments in 1D models have been obtained by means of the following approaches:

1. The introduction of shear correction factors.

2. The use of warping functions based on the de SaintVenant solution.

3. The variational asymptotic solution (VABS).

4. Generalized beam theories (GBT).

5. Higher-order beam models.

A considerable amount of work has been done to try to improve the global response of classical beam theories through the use of appropriate shear correction factors, as in the books by Timoshenko [11] and Sokolnikoff [12]. Many interesting articles [13-17] have been published on this topic.

ElFatmi[18] introducedimprovements of the displacement models over the beam section by introducing a warping function to enhance the description of the normal and shear stress of the beam. End effects due to boundary conditions have been investigated by means of this model [19].

The de Saint-Venant solution has been the theoretical framework of many advanced beam models. 3D elasticity equations were reduced to beam-like structures [20]. The resulting solution was modeled as the sum of a de SaintVenant part and a residual part, and applied to high-aspectratio beams with thin-walled sections. Other beam theories have been based on the displacement field proposed [21] and solved by means of semi-analytical finite elements as in [22].

Asymptotic-type expansions have been proposed in [23] on the basis of variational methods. That work represented the starting point of an alternative approach to constructing refined beam theories where a characteristic parameter (e.g. the cross-sectional thickness of a beam) is exploited to build an asymptotic series. Those terms that exhibit the same order of magnitude as the parameter when it vanishes are retained. Some valuable contributions on asymptotic methods are those related to VABS [24-26]. The key feature of this methodology is that the 1D model is governed by variationally consistent and geometrically exact governing equations which provide asymptotically exact stress and strain recovery by means of a beam model having a low number of degrees of freedom. Regular and thin-walled beams can be accounted for.

Generalized beam theories (GBT) have been derived from Schardt's work [27]. GBT enhances classical theories by exploiting piece-wise beam descriptions of thin-walled sections. It has been extensively employed and extended in various forms [28]. In the GBT framework, the cross-section displacement field of a thin-walled beam is assumed as a linear combination of deformation modes defined on a number of cross-section nodes. The proper choice of the number of modes depends on the cross-section type and the number of fold lines [29].

Many other higher-order theories which are based on enhanced displacement fields over the beam cross-section have been introduced to include non-classical effects. Some considerations on higher order beam theories were made by Washizu [30]. An advanced model was proposed in [31] where classical finite beam elements were improved by introducing new degrees of freedom to describe the crosssection behavior. Other refined beam models can be found in the excellent reviews by Kapania and Raciti [32-33], which focused on: bending, vibration, wave propagations, buckling and post-buckling. Excellent papers on structural dynamic and aeroelastic problems of thin-walled structures by means of higher-order beams are those by Librescu [34-36].

The aim of this work is to present aeroelastic models based on highly accurate structural models and low-order aerodynamic tools. Particular attention was given to the flutter of wings and thin-walled panels. The adoption of the present formulation allows one to predict flutter with remarkable reductions of computational costs and acceptable accuracy.

This work is embedded in the framework of the Carrera Unified Formulation (CUF) for higher-order 1D models [37]. CUF had been initially developed for plates and shells [38-39], and more recently for beams [40-41]. The unique contribution given by CUF models is due to their hierarchical capabilities which make the choice of the expansion functions $\left(\mathrm{F}_{\tau}\right)$ and their order arbitrary. This means that anyorder structural models can be implemented with no need for formal changes in the problem equations and matrices. CUF can therefore deal with arbitrary geometries, boundary conditions and material characteristics with no need for ad hoc formulations.

Static [41-43], free-vibration [44-46] and buckling [4748] analyses have shown the enhanced capabilities of CUF 1D models, which are able to detect shell- and solid-like solutions for different structural models, including thinwalled models under point loads and shell-like natural modes. A further extension of the present formulation [49- 
51] dealt with open cross-sections, boundary conditions enforced on lateral edges and component-wise approaches.

Varello et al. [52] extended CUF 1D to steady aeroelasticity by using the Vortex Lattice Method (VLM). In this work, unsteady aeroelasticity is considered through the Doublet Lattice Method(DLM). CUF was exploited to derive structural refined finite $1 \mathrm{D}$ elements; that is, the structural component of the proposed advanced aeroelastic formulation was based on 1D CUF higher-order models. This paper is organized as follows: first, the structural and aeroelastic formulations are briefly described; afterwards, results and their discussion are presented; eventually, main conclusions are drawn.

\section{Structural Formulation: CUF 1D Models}

The unified formulation of the beam cross-section displacement field is described by an expansion of generic functions, $F_{\tau}$,

$$
\boldsymbol{u}=F_{\tau} \boldsymbol{u}_{\tau}, \quad \tau=1,2, \ldots, M
$$

where $\mathrm{F}_{\tau}$ are functions of the cross-section coordinates $x$ and $z, \mathbf{u}_{\tau}$ is the displacement vector, and $M$ stands for the number of terms of the expansion. According to the Einstein notation, the repeated subscript $\tau$ indicates summation. The choice of $\mathrm{F}_{\tau}$ and $M$ is arbitrary, that is, different base functions of any order can be taken into account to model the displacement field of a beam above its cross-section. In this work, Taylor-like polynomial expansions $\left(x^{i} z^{j}\right)$ of the displacement field above the cross-section of the structure were used, where $i$ and $j$ are positive integers. The order $N$ of the expansion is arbitrary and is set as an input of the analysis. The choice of $N$ for a given structural problem is usually made through a convergence study. For example, the second-order model, $N=2$, is based on the following displacement field:

$$
\begin{aligned}
& u_{x}=u_{x_{1}}+x u_{x_{2}}+z u_{x_{3}}+x^{2} u_{x_{4}}+x z u_{x_{5}}+z^{2} u_{x_{6}} \\
& u_{y}=u_{y_{1}}+x u_{y_{2}}+z u_{y_{3}}+x^{2} u_{y_{4}}+x z u_{y_{5}}+z^{2} u_{y_{6}} \\
& u_{z}=u_{z_{1}}+x u_{z_{2}}+z u_{z_{3}}+x^{2} u_{z_{4}}+x z u_{z_{5}}+z^{2} u_{z_{6}}
\end{aligned}
$$

The 1D model described by Eq. 2 has 18 generalized displacement variables: three constant, six linear, and nine parabolic terms.

The governing equations were derived by means of the Principle of Virtual Displacements (PVD). Starting from the unified form of the displacement field in Eq. 1, stiffness, mass, and loading arrays can be obtained in terms of fundamental nuclei whose form is independent of the order of the model. The finite element method (FEM) was adopted to overcome the limits of analytical solutions in terms of geometry, loading, and boundary conditions. The displacement variables are interpolated along the axis of the beam by means of the shape functions, $N_{i}$,

$$
\boldsymbol{u}=N_{i} F_{\tau} \boldsymbol{q}_{\tau i}
$$

where $\boldsymbol{q}_{\mathrm{i}}$ is the nodal displacement vector. Beam elements with four (B4) nodes were considered in this paper. It is important to emphasize that the beam model order is given by the expansion on the cross-section, whereas the number of nodes per each element is related to the approximation along the longitudinal axis of the beam $(y)$. An $N$-order beam model is therefore a theory that exploits an $\mathrm{N}$-order Taylorlike polynomial to describe the kinematics of the beam cross-section.

According to the principle of virtual displacements,

$$
\delta L_{\text {int }}=\int_{V}\left(\delta \boldsymbol{\varepsilon}^{T} \boldsymbol{\sigma}\right) d V=\delta L_{\text {ext }}
$$

where $L_{\text {int }}$ stands for the strain energy, $L_{\text {ext }}$ is the work of the external loadings, $\delta$ stands for the virtual variation, $\boldsymbol{\sigma}$ is the stress vector and $\boldsymbol{\varepsilon}$ is the strain vector. A compact form of the virtual variation of the strain energy can be obtained as shown in [37],

$$
\delta L_{i n t}=\delta \boldsymbol{q}_{\tau i}^{T} \boldsymbol{K}^{i j \tau s} \boldsymbol{q}_{s j}
$$

where $\boldsymbol{K}^{i j s}$ is the stiffness matrix written in the form of the fundamental nuclei. Superscripts indicate the four indexes exploited to assemble the matrix: $i$ and $j$ are related to the shape functions, and $\tau$ and $s$ are related to the expansion functions. The fundamental nucleus is a $3 \times 3$ array which is formally independent of the order of the beam model. In compact notation, the stiffness matrix for a given material property set can be written as:

$$
\begin{aligned}
& \mathbf{K}^{i j \tau s}= \\
& I_{l}^{i j} \triangleleft\left(\mathbf{D}_{n p}^{T} F_{\tau} \mathbf{I}\right)\left[\tilde{\mathbf{C}}_{n p}\left(\mathbf{D}_{p} F_{s} \mathbf{I}\right)+\tilde{\mathbf{C}}_{n n}\left(\mathbf{D}_{n p} F_{s} \mathbf{I}\right)\right]+ \\
& \left(\mathbf{D}_{p}^{T} F_{\tau} \mathbf{I}\right)\left[\tilde{\mathbf{C}}_{p p}\left(\mathbf{D}_{p} F_{S} \mathbf{I}\right)+\tilde{\mathbf{C}}_{p n}\left(\mathbf{D}_{n p} F_{s} \mathbf{I}\right)\right] \triangleright_{\Omega}+ \\
& I_{l}^{i j, y} \triangleleft\left[\left(\mathbf{D}_{n p}^{T} F_{\tau} \mathbf{I}\right) \tilde{\mathbf{C}}_{n n}+\left(\mathbf{D}_{p}^{T} F_{\tau} \mathbf{I}\right) \tilde{\mathbf{C}}_{p n}\right] F_{s} \triangleright_{\Omega} \mathbf{I}_{\Omega y}+ \\
& I_{l}^{i, y j} \mathbf{I}_{\Omega y} \triangleleft F_{\tau}\left[\tilde{\mathbf{C}}_{n p}\left(\mathbf{D}_{p} F_{s} \mathbf{I}\right)+\tilde{\mathbf{C}}_{n n}\left(\mathbf{D}_{n p} F_{s} \mathbf{I}\right)\right] \triangleright_{\Omega}+ \\
& I_{l}^{i, y j, y} \mathbf{I}_{\Omega y} \triangleleft F_{\tau} \tilde{\mathbf{C}}_{n n} F_{s} \triangleright_{\Omega} \mathbf{I}_{\Omega y}
\end{aligned}
$$

where

$$
\begin{aligned}
& \mathbf{I}_{\Omega y}=\left[\begin{array}{lll}
0 & 1 & 0 \\
1 & 0 & 0 \\
0 & 0 & 1
\end{array}\right] \triangleleft \ldots \triangleright_{\Omega}=\int_{\Omega} \ldots d \Omega \\
& \left(I_{l}^{i j}, I_{l}^{i j, y}, I_{l}^{i, y}, I_{l}^{i, y j, y}\right) \\
& \quad=\int_{l}\left(N_{i} N_{j}, N_{i} N_{j, y}, N_{i, y} N_{j}, N_{i, y} N_{j, y}\right) d y
\end{aligned}
$$


$\tilde{\mathbf{C}}$ is the material coefficient matrix and $\mathbf{D}$ is the differential operator matrix. For the sake of brevity, their expressions are not reported here, but they can be found in [37]. The following about the formal expression of $\boldsymbol{K}^{i j \tau s}$ should be emphasized:

1. That it does not depend on the expansion order,

2. Nor does it depend on the choice of the $F_{\tau}$ expansion polynomials.

These are key points of CUF which permit the implementation of any order of multiple class theories, with only nine FORTRAN statements.

The virtual variation of the work of inertial loadings is

$$
\delta L_{\text {ine }}=\int_{V} \rho \ddot{\boldsymbol{u}} \delta \boldsymbol{u}^{T} d V
$$

where $\rho$ stands for the density of the material, and $\ddot{\boldsymbol{u}}$ is the acceleration vector. Equation 9 can be rewritten in a compact manner as follows:

$$
\delta L_{i n e}=\delta \boldsymbol{q}_{\tau i}^{T} \boldsymbol{M}^{i j \tau s} \ddot{\boldsymbol{q}}_{s j}
$$

where $\boldsymbol{M}^{i j \tau s}$ is the mass matrixin the form of the fundamental nucleus whose components can be found in [50].

The undamped dynamic problem can be written as follows:

$$
M \ddot{a}+K a=p
$$

where $\boldsymbol{a}$ is the vector of the nodal unknowns and $\mathrm{p}$ is the loading vector. Introducing harmonic solutions, it is possible to compute the natural frequencies, $\Omega_{i}$, for the homogenous case, by solving an eigenvalue problem,

$$
\left(-\Omega_{i}^{2} \boldsymbol{M}+\boldsymbol{K}\right) \boldsymbol{\phi}_{i}=0
$$

where $\varphi_{i}$ is the $i$-th eigenvector.

\section{Aeroelastic Formulation: Doublet Lattice Method and Mesh-to-Mesh Transforma- tions}

Following Landahl [53] or Albano [5], the normalwash at a point with coordinates $x, y$ due to the pulsating pressure jump $\overline{\Delta p}$ in the point $\xi, \eta$ has the following expression:

$$
\bar{w}=\frac{1}{8 \pi} \int_{A} \overline{\Delta p} K\left(x_{0}, y_{0}, \omega, M\right) d A
$$

where $M$ is the Mach number, $\omega$ is the circular frequency and

$$
x_{0}=x-\xi ; \quad y_{0}=y-\eta
$$

The kernel function $(K)$ formal expression is not reported here for the sake of brevity, but can be found in [53]. Eq. 13 can be numerically solved by means of the doublet lattice method (DLM). In the DLM framework, a lifting surface is discretized in a number of panels, and the following algebraic system of equations has to be solved:

$$
\bar{w}_{i}=\sum_{j=1}^{N_{A P}} D_{i j} \overline{\Delta p}_{j}
$$

where $N_{A P}$ indicates the total number of aerodynamic panels and $D_{i j}$ is the normal wash factor. In this paper, $D_{i j}$ was calculated by exploiting Rodden's quartic DLM [6]. For the sake of brevity, the procedure to compute the normalwash factor is not reported here, but it can be found in Rodden's paper. It is important to emphasize that the steady contribution to $D_{i j}$ was computed via the vortex lattice method (VLM) [54].

The unsteady aeroelastic analysis was carried out by considering a set of modal shapes as generalized motions for the unsteady aerodynamic generalized force generation. Each set of modal shapes, $\varphi_{m}$, was defined on a set of points above the structure. Slopes and displacements at control and load points of the aerodynamic panels are then given by

$$
\begin{aligned}
& \frac{\partial \boldsymbol{Z}_{\mathrm{m}}}{\partial x}=\boldsymbol{A} \cdot \boldsymbol{\phi}_{m} \\
& \widetilde{\boldsymbol{Z}}_{\mathrm{m}}=\widetilde{\boldsymbol{A}}^{*} \cdot \boldsymbol{\phi}_{m} \\
& \boldsymbol{Z}_{\mathrm{m}}=\boldsymbol{A}^{*} \cdot \boldsymbol{\phi}_{m}
\end{aligned}
$$

where $\boldsymbol{A}, \tilde{\boldsymbol{A}}^{*}$ and $\boldsymbol{A}^{*}$ are computed through the Infinite Plate Spline (IPS) [55]. For the sake of brevity, the explicit expressions of these matrices are not reported here, but they can be found in [56]. IPS was chosen in order to better exploit the shell-like capabilities of the present 1D structural formulation, as shown by Varello et al. [52]. Under the assumption of simple harmonic motion, it is possible to demonstrate that the vector that contains the normalized (using the velocity $V_{\infty}$ parallel to $x$ ) normalwash has the following expression (the boundary condition is enforced on all control points of the lifting surface):

$$
\mathbf{w}_{m}=i \frac{\omega}{V_{\infty}} \boldsymbol{Z}_{\mathrm{m}}+\frac{\partial z_{\mathrm{m}}}{\partial x}
$$

where all the vector quantities have to be understood as vectors of amplitudes of the harmonic motion, and $i$ is the imaginary unit.

\section{Generalized Matrices and G-Method}

The generalized aerodynamic matrix for a given reduced frequency $(k)$ is given by 
$Q_{i j}(i k)=\sum_{N=1}^{N_{A P}} \Delta p_{j}^{N}(i k) \tilde{Z}_{\mathrm{i}}^{N} A^{N}$

where:

- $\quad k=\omega b / L, b$ is the reference length (equal to the half of the reference chord) and $L$ is the length of the structure.

- $\Delta p_{j}^{N}(i k)$ is the pressure jump due to the $\mathrm{j}$-th set of motions (modal shapes), acting on the $\mathrm{N}$-th aerodynamic panel and evaluated for a given reduced frequency. The computation of the pressure jump is performed by means of the DLM.

- $\tilde{Z}_{\mathrm{i}}^{N}$ is the $i$-th motion set evaluated at the $N$-th aerodynamic panel. Starting from the $i$-th modal shape given by a structural model, the $i$-th motion set is then mapped on the aerodynamic panels by means of the splining process. In this work, modal shapes were evaluated by means of CUF 1D models.

- $A^{N}$ is the area of the $N$-th panel.

- $\mathbf{Q}(i k)$ is a square matrix with $N_{\text {modes }} \times N_{\text {modes }}$ elements, where $N_{\text {modes }}$ indicates the total number of natural modes adopted. Typically, $N_{\text {modes }}$ ranges from 10 to 20.

The generalized mass matrix is given by

$$
\widetilde{\boldsymbol{M}}=\boldsymbol{\phi}^{T} \boldsymbol{M} \boldsymbol{\phi}
$$

where:

- $\varphi$ is a matrix containing a given number of modal shapes, dimension: $N_{D O F} \times N_{\text {modes }} . N_{D O F}$ is the total number of DOFs of the structural model.

- $\quad \boldsymbol{M}$ is the mass matrix of the structure (dimension: $\left.N_{D O F} \times N_{D O F}\right), M$ is a square diagonal matrix with $N_{\text {modes }} \times N_{\text {modes }}$ terms.

- The generalized stiffness matrix is a square diagonal $\left(N_{\text {modes }} \times N_{\text {modes }}\right)$ matrix, and its diagonal terms are given by

$\widetilde{K}_{i i}=\omega_{i}^{2} \widetilde{M}_{i i}$

where $\omega_{i}$ is the oscillatory frequency associated with the i-th modal shape.

The g-method was introduced by Chen [57] and it is based on a damping perturbation technique and a firstorder model of the damping term. Its derivation exploits the aerodynamics in the Laplace domain and can be found in [57]. The basic assumption of the g-method is based on the following approximation of the generalized aerodynamic matrix:

$$
\widetilde{\boldsymbol{Q}}(p) \approx \widetilde{\boldsymbol{Q}}(i k)+g \widetilde{\boldsymbol{Q}}^{\prime}(i k), \text { for } g \ll 1
$$

where $g=\gamma k$ and $\gamma$ is the transient decay rate coefficient. Eq. 23 leads to the g-method equation:

$$
\left[\left(\frac{V_{\infty}}{b}\right)^{2} \widetilde{\boldsymbol{M}} p^{2}+\widetilde{\boldsymbol{K}}-\frac{1}{2} \rho V_{\infty}^{2} \widetilde{\boldsymbol{Q}}^{\prime}(i k) g-\frac{1}{2} \rho V_{\infty}^{2} \widetilde{\boldsymbol{Q}}(i k)\right]\{\boldsymbol{q}(p)\}=0
$$

Where $p$ is the nondimensional Laplace parameter $(p=g$ $+i k)$

The generalized aerodynamic matrix, $\tilde{Q}(i k)$, is provided by the unsteady aerodynamic model (DLM) in the frequency domain. The computation of $\tilde{Q}^{\prime}(i k)$ has to be performed numerically. A central difference scheme can be used. with a forward scheme at $k=0$. Three new matrices are introduced,

$$
\begin{aligned}
& \boldsymbol{A}=\left(\frac{V_{\infty}}{b}\right)^{2} \widetilde{\boldsymbol{M}} \\
& \boldsymbol{B}=2 i k\left(\frac{V_{\infty}}{b}\right)^{2} \widetilde{\boldsymbol{M}}-\frac{1}{2} \rho V_{\infty}^{2} \widetilde{\boldsymbol{Q}}^{\prime}(i k) \\
& \boldsymbol{C}=-k^{2}\left(\frac{V_{\infty}}{b}\right)^{2} \widetilde{\boldsymbol{M}}+\widetilde{\boldsymbol{K}}-\frac{1}{2} \rho V_{\infty}^{2} \widetilde{\boldsymbol{Q}}(i k)
\end{aligned}
$$

Eq. 24 becomes

$$
\left[g^{2} \boldsymbol{A}+g \boldsymbol{B}+\boldsymbol{C}\right]\{\boldsymbol{q}\}=0
$$

This is a second-order linear system in $g$, and the g-method aims to find those solutions having $\operatorname{Im}(g)=0$. Eq. 26 is rewritten in the state-space form,

$$
[\boldsymbol{D}-g \boldsymbol{I}]\{\boldsymbol{X}\}=0
$$

where

$$
D=\left[\begin{array}{cc}
0 & I \\
-A^{-1} C & -A^{-1} B
\end{array}\right]
$$

A so-called reduced-frequency-sweep technique is adopted to find the solution having $\operatorname{Im}(g)=0$ :

- a range of $k$ values is chosen, [0, $k_{\max }$, with $k_{\max }$ as the highest value in the reduced frequency list of the unsteady aerodynamic computation;

- $\quad$ a step $\Delta k$ is fixed;

- $\quad$ at each step $i$ the eigenvalues of $\boldsymbol{D}$ are computed for $k_{i}=k_{i-1}+\Delta k$;

- a sign change of the imaginary part of each eigenvalue is searched for;

- $\quad$ if a sign change occurs and $\operatorname{Re}(g)>0$, the reduced flutter frequency will be computed by means of a linear interpolation. 


\section{Results and Discussion}

Numerical examples are presented and discussed in this section. After a preliminary aerodynamic assessment, flutter analyses were carried out for the following structural configurations:

1. Single- and double-swept wings.

2. A panel with the leading edge clamped.

Results from open literature and experiments were exploited for comparison purposes. All results were obtained in the case of incompressible flow $(M=0)$.

\subsection{Preliminary doublet lattice method assessment: lift distribution of a wing with oscillating flaps}

A wing with oscillating outer flaps is considered in this section. This model was retrieved from [58] and its geometry

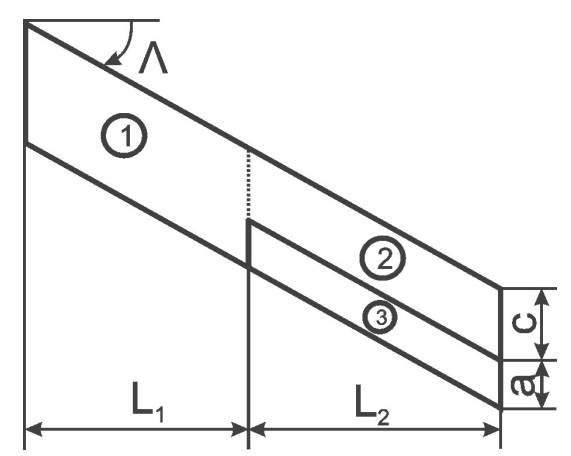

Fig. 1. Wing model with outer flap
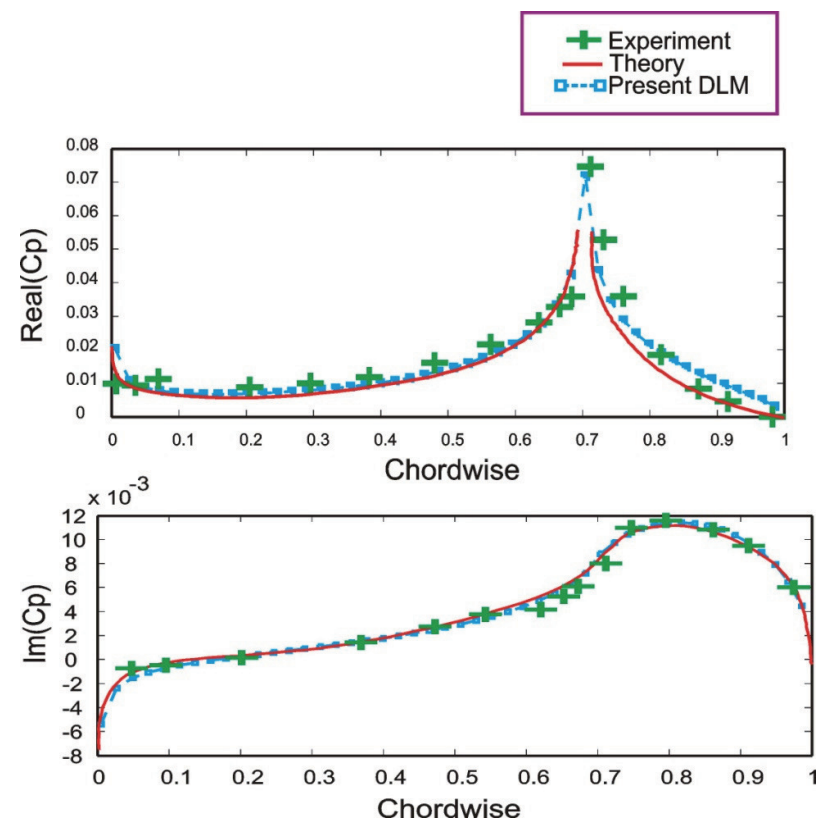

Fig. 2. Chordwise pressure distribution at $y / L=0.69$ is shown in Fig. 1 (only half wing is shown), where: $L=L_{1}+$ $L_{2}=0.88 \mathrm{~m}\left(L_{1}=0.41 \mathrm{~m}, L_{2}=0.47 \mathrm{~m}\right), c=0.42 \mathrm{~m}$ and $a=0.18$ $\mathrm{m}$. The maximum flap oscillation angle is equal to $0.66^{\circ}$ and the reduced oscillatory frequency, $k$, is equal to 0.372 . The half wing was divided into three different portions, namely 1, 2 and 3; different aerodynamic meshes were adopted for each portion. Table 1 shows each mesh set adopted in terms of chordwise $\times$ spanwise aerodynamic panels.

Table 2 presents lift coefficients, $C_{L}$, by means of the present quartic DLM and the Doublet Point Method (DPM) from [59]. Fig. 2 shows chordwise pressure coefficient distributions with comparisons between experimental data from [58] and analytical results from [60]. It can be stated that a general excellent agreement was found between the present DLM and the experimental and analytical results.

\subsection{Swept wing}

Flutter analyses of swept wings were considered in this

Table 1. Mesh sets adopted for the wing model with oscillating flaps

\begin{tabular}{cccc}
\hline Mesh Set & Portion 1 & Portion 2 & Portion 3 \\
\hline 1 & $6 \times 3$ & $4 \times 4$ & $2 \times 4$ \\
2 & $12 \times 6$ & $8 \times 8$ & $4 \times 8$ \\
3 & $24 \times 12$ & $16 \times 16$ & $8 \times 16$ \\
4 & $48 \times 24$ & $32 \times 32$ & $16 \times 32$ \\
\hline
\end{tabular}

Table 2. $C_{L}$ coefficient for different mesh sets

\begin{tabular}{cc}
\hline Mesh Set & $C_{L} \times 10^{3}$ \\
\hline 1 & $9.262+1.822 i$ \\
2 & $9.210+1.818 i$ \\
3 & $9.181+1.808 i$ \\
4 & $9.169+1.804 i$ \\
DPM [59], Mesh set 1 & $9.5+1.8 i$ \\
\hline
\end{tabular}

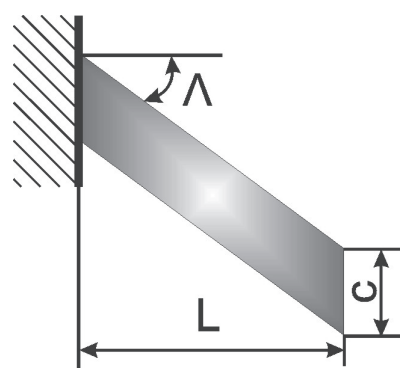

Fig. 3. Swept wing geometry, the sweep angle shown is positive 
section. The swept wing model was retrieved from [61] where plate models based on the Classical Laminated Theory (CLT) were used for flutter analyses. The wing model investigated has the following characteristics (see Fig. 3): $L=0.305 \mathrm{~m}, c$ $=0.076 \mathrm{~m}$, thickness, $t=0.001 \mathrm{~m}$. The material is isotropic with $E=73.8 \mathrm{GPa}, G=27.6 \mathrm{GPa}$ and $\rho=2768 \mathrm{~kg} / \mathrm{m}^{3}$. An $8 \times$ 30 aerodynamic mesh was adopted, which was chosen via a convergence study.

Table 3 presents the first three natural frequencies of a backward swept configuration. Unless otherwise indicated, the frequencies reported are related to bending modes. The results from different 1D structural models are shown, including classical models (EBBT and TBT) The indication of the number of DOFs of each structural model is reported

Table 3. Effect of the CUF 1D expansion order (N) on vibration frequencies, $\mathrm{Hz}, \Lambda=30^{\circ}, 20 \mathrm{~B} 4$ mesh

\begin{tabular}{lcccc}
\hline Model & $f_{1}$ & $f_{2}$ & $f_{3}$ & DOFs \\
\hline EBBT & 8.967 & 56.192 & 157.335 & 183 \\
TBT & 8.966 & 56.189 & 157.320 & 305 \\
$N=1$ & 8.966 & 56.185 & 157.308 & 549 \\
$N=2$ & 7.199 & 44.462 & $97.939^{*}$ & 1098 \\
$N=3$ & 7.125 & 43.778 & $97.939^{*}$ & 1830 \\
$N=4$ & 7.093 & 43.529 & $73.296^{*}$ & 2745 \\
\hline$*$ torsional mode & & &
\end{tabular}
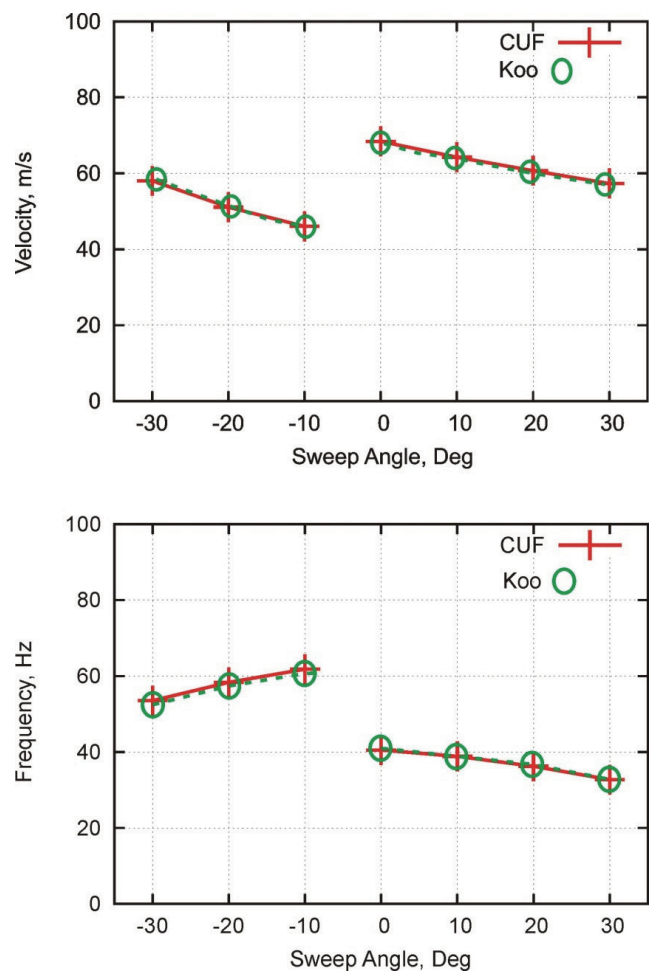

Fig. 4. Effect of the sweep angle on flutter conditions in the last column. Convergence studies are presented in Tables 4 and 5, where the effects of the structural mesh and the Taylor expansion order on flutter conditions are reported, respectively. The flutter conditions for different sweep angles are given in Table 6 and Fig. 4, where the results from the present formulation are compared with those by Koo [61]. The analysis of the results suggests the following:

1. Higher-order 1D models are mandatory in order to predict torsional modes and flutter conditions of wings.

2. The proper detection of torsional modes requires at least an $N=3$ model, as also shown in [37].

3. For these wing configurations, the first torsional mode plays a fundamental role in flutter conditions. This means that the proper detection of flutter conditions requires at least a third-order $1 \mathrm{D}$ structural model $(N$ $=3)$.

4. Flutter predictions by the present structural formulation (1D) are in excellent agreement with those by Koo (CLT, 2D).

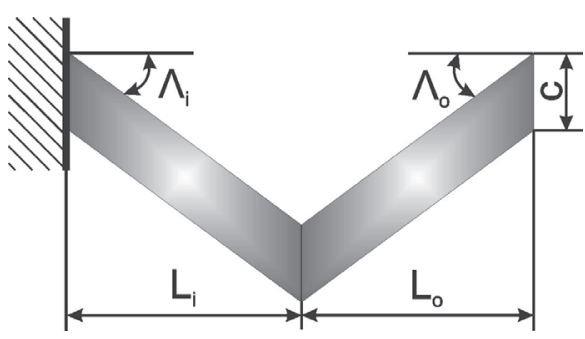

Fig. 5. Double-swept wing geometry, the depicted inbound sweep angle is positive, the outbound angle is negative

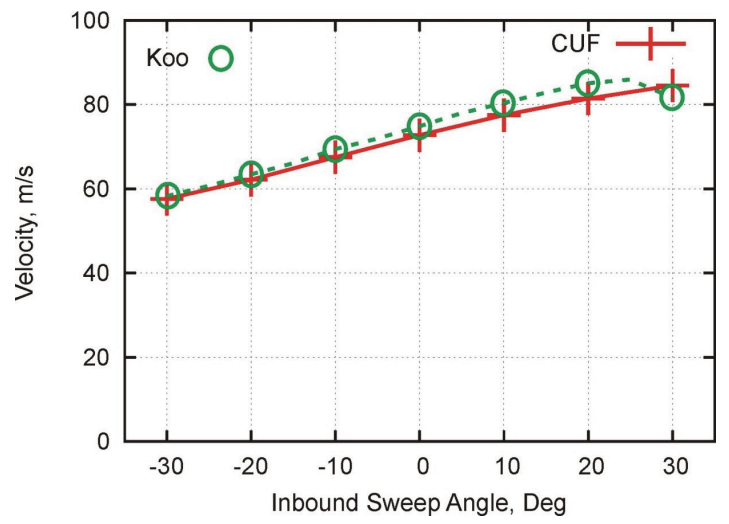

Fig. 6. Effect of the inbound sweep angle on flutter conditions

Table 4. Effect of the structural mesh on flutter conditions, $\mathrm{N}=3$, $\Lambda=30^{\circ}$

\begin{tabular}{lrcc}
\hline Mesh & Velocity, $\mathrm{m} / \mathrm{s}$ & Frequency, $\overrightarrow{H z}$ \\
\hline 20 & B4 & 59.202 & 53.888 \\
30 & B4 & 59.456 & 53.688 \\
\hline
\end{tabular}


5. Very low computational costs were required.

\subsection{Double-Swept wing}

A double-swept wing was considered in this section. This model was retrieved from [61]. Wing characteristics are described in Fig. 5, and their values are the following: $L_{i}+L_{0}=$ $0.305 \mathrm{~m}, L_{i}=L_{o}, C=0.076 \mathrm{~m}$, thickness, $t=0.001 \mathrm{~m}, \Lambda_{o}=-30^{\circ}$. The inbound sweep angle, $\Lambda_{i}$, was assumed to range from $-30^{\circ}$ to $+30^{\circ}$. An isotropic material was considered $(E=73.8$ GPa, $\left.G=27.6 \mathrm{GPa}, \rho=2768 \mathrm{Kg} / \mathrm{m}^{3}\right)$. An $8 \times 20$ aerodynamic mesh was adopted fir each wing segment (320 panels), and a

Table 5. Effect of the CUF 1D expansion order, N, on flutter conditions, 20 B4 mesh

\begin{tabular}{llll}
\hline Model & Velocity, $\mathrm{m} / \mathrm{s}$ & Frequency, Hz & DOFs \\
\hline $\mathrm{N}=2$ & 84.206 & 67.053 & 1089 \\
$\mathrm{~N}=3$ & 59.202 & 53.888 & 1830 \\
$\mathrm{~N}=4$ & 58050 & 53.490 & 2745 \\
\hline
\end{tabular}

Table 6. Flutter conditions for different sweep angles, 20 B4 mesh, $\mathrm{N}=4$

\begin{tabular}{crc}
\hline$\Lambda$ & Velocity, $\mathrm{m} / \mathrm{s}$ & Frequency, $\mathrm{Hz}$ \\
\hline$-30^{\circ}$ & 58.050 & 53.490 \\
$-20^{\circ}$ & 51.109 & 58.339 \\
$-10^{\circ}$ & 46.029 & 61.832 \\
$0^{\circ}$ & 68.406 & 40.493 \\
$+10^{\circ}$ & 64.262 & 38.865 \\
$+20^{\circ}$ & 60.684 & 36.231 \\
$+30^{\circ}$ & 57.339 & 32.680 \\
\hline
\end{tabular}

$20 \mathrm{~B} 4$ mesh was used for the structural discretization. These meshes were chosen via a convergence study.

Table 7 shows the effect of the Taylor expansion order on flutter conditions for $\Lambda_{i}=30^{\circ}$. All the other inbound sweep angle configurations were considered in Table 8 and Fig. 6 , where results from the present model and from Koo's paper are shown.

The following statements hold:

1. As previously shown for the single-swept configuration, at least an $\mathrm{N}-3$ model is required to compute flutter conditions.

2. Very good agreement was found with results based on plate models.

3. It can be concluded that the present $1 \mathrm{D}$ structural formulation can properly detect the flutter of double-

Table 7. Effect of the expansion order on flutter conditions of the double swept wing, $\Lambda_{\mathrm{i}}=30^{\circ}$

\begin{tabular}{ccc}
\hline Model & Velocity, $\mathrm{m} / \mathrm{s}$ & Frequency, $\mathrm{Hz}$ \\
\hline $\mathrm{N}=2$ & 89.467 & 64.899 \\
$\mathrm{~N}=3$ & 85.917 & 55.738 \\
$\mathrm{~N}=4$ & 84.537 & 55.403 \\
\hline
\end{tabular}

Table 8. Flutter conditions for different inbound sweep angles, $\mathrm{N}=4$

\begin{tabular}{ccc}
\hline$\Lambda_{\mathbf{i}}$ & Velocity, m/s & Frequency, Hz \\
\hline$-20^{\circ}$ & 62.161 & 56.913 \\
$-10^{\circ}$ & 67.524 & 58.777 \\
$0^{\circ}$ & 72.757 & 59.227 \\
$+10^{\circ}$ & 77.510 & 58.923 \\
$+20^{\circ}$ & 81.443 & 57.481 \\
\hline
\end{tabular}

Table 9. Effect of the CUF 1D expansion order, $\mathrm{N}$, on vibration frequencies, $\mathrm{Hz}$, flutter velocity, $\mathrm{m} / \mathrm{s}$, and flutter frequency, $\mathrm{Hz}$, of the cantilever plate, $20 \mathrm{~B} 4$ mesh

\begin{tabular}{lcccccc}
\hline Model & $f_{1}$ & $f_{2}$ & $f_{3}$ & $V_{\mathrm{F}}$ & $f_{\mathrm{F}}$ & DOFs \\
\hline EBBT & 6.346 & 39.770 & 111.356 & 26.867 & 34.779 & 138 \\
TBT & 6.346 & 39.769 & 111.352 & 26.866 & 34.779 & 230 \\
$N=1$ & 6.346 & 39.769 & 111.352 & 26.866 & 34.779 & 414 \\
$N=2$ & 6.734 & $16.842^{*}$ & 42.202 & 28.470 & 37.420 & 828 \\
$N=3$ & 6.671 & $16.189^{*}$ & 40.753 & 28.801 & 35.785 & 1380 \\
$N=4$ & 6.667 & $16.100^{*}$ & 40.678 & 28.779 & 35.796 & 2070 \\
{$[62]($ Exp) } & 7.8 & - & - & 27.43 & 40.2 & - \\
{$[63]($ CLT) } & 6.777 & 42.003 & 101.14 & 29.5 & 36.003 & - \\
\hline${ }^{*}$ torsional mode & \multicolumn{7}{c}{} \\
\hline
\end{tabular}


swept wing models.

\subsection{Panel flutter}

This section was focused on the flutter analysis of a square panel clamped along its leading edge and free on the other three edges. This model was retrieved from and [62]. [63] The plate has a thickness of 0.020 inches and a length of 10 inches. An isotropic material was adopted $(E=68.9 \mathrm{GPa}, v=$ $\left.0.33, \rho=2700 \mathrm{Kg} / \mathrm{m}^{3}\right)$. A $40 \times 8$ aerodynamic mesh and a 20 B4 structural mesh were adopted.

Table 9 shows natural frequencies and flutter conditions from different models and experimental results. Unless otherwise indicated, natural frequencies are related to bending modes. It is important to underline the following:

1. Lower-order models are able to quite accurately describe bending, whereas higher-order models are needed for torsion.

2. Since the second bending mode is involved in flutter, lower-order models can predict the instability in this case.

3. Results from the present structural 1D formulation are in good agreement with those from experiments and CLT; larger differences with the experimental data were observed in the detection of the frequencies. However, it is important to emphasize that in [62], the calculated natural frequencies were also provided, and they were equal to 6.7 and $42 \mathrm{~Hz}$, respectively. Calculated flutter frequencies were also provided, and depending on different aerodynamic models, they ranged between 37.5 and $42.6 \mathrm{~Hz}$.

\section{Conclusion}

Flutter analyses of isotropic lifting surfaces have been carried out in this study. The aeroelastic formulation exploited in this work was based on 1D advanced structural models and the doublet lattice method. The aerodynamic and structural mesh coupling has been conducted through the infinite plate spline method. Flutter conditions have been computed via the g-method.

The advanced structural models have been developed in the framework of the Carrera Unified Formulation (CUF). CUF 1D models exploit Taylor-like polynomials to define the displacement field above the cross-section of the beam. Anyorder structural models can be implemented since the order of the Taylor expansion is a free parameter of the formulation. This means that the order of the structural model can be set as an input, and convergence studies have to be carried out in order to establish the proper theory order necessary for a given problem.

The analyses conducted suggest the following:

1. Refined 1D structural models are mandatory for proper flutter analyses of lifting surfaces.

2. Refinements are needed to capture torsional modal shapes and to deal with thin walls.

3. The present $1 \mathrm{D}$ structural model is cost-competitive if compared to $2 \mathrm{D}$ plate models, with no accuracy losses.

The use of CUF 1D could offer even greater advantages in the fluid-structure-interaction analysis of flexible structures with highly deformable cross-section, such as adaptive wings or arteries. CUF 1D can, in fact, predict in-plane distortion of thin-walled structures with high accuracy and very low computational costs, as shown in [37]. Future works should deal with the coupling of CUF 1D with computational fluid dynamics tools.

\section{Acknowledgements}

The financial support of the Italian Fulbright Commission and of the European Project SARISTU is gratefully acknowledged. The author warmly thanks Dr. Luciano Demasi (San Diego State University) and Prof. Erasmo Carrera (Politecnico di Torino) for their helpful efforts in helping him in the research activity related to this paper.

\section{References}

[1] Yurkovich, R., "Status of unsteady aerodynamic prediction for flutter of high-performance aircraft", Journal of Aircraft, Vol. 40, No. 5, 2003, pp. 832--842.

[2] Schuster, D.M., Liu, D.D., and Huttsell L.J., "Computational aeroelasticity: success, progress, challenge", Journal of Aircraft, Vol. 40, No. 5, 2003, pp. 843--856.

[3] Theodorsen, T., and Garrick, I.E., "A theoretical and experimental investigation of the flutter problem", NACA, City, State or Country, 1940,

[4] Yates, E.C., "Modified-strip-analysis method for predicting wing flutter at subsonic to hypersonic speeds", Journal of Aircraft, Vol. 3, No. 1, 1966, pp. 25--29.

[5] Albano, E., and Rodden, W.P., "A doublet-lattice method for calculating lift distributions on oscillating surfaces in subsonic flows", AIAA Journal, Vol. 7, No. 2, 1969, pp. 279-285.

[6] Rodden, W.P., Taylor, P.F., and McIntosh, S.C.J., "Further Refinement of the Subsonic Doublet-Lattice Method,"Journal of Aircraft, Vol. 33, No. 5, 1998, pp. 720--726. 
[7] Bernoulli, D., "De vibrationibus et sono laminarum elasticarum. In: Commentarii Academiae Scientiarum Imperialis Petropolitanae", Petropoli, 1751.

[8] Euler, L., De curvis elasticis Lausanne and Geneva, Bousquet, 1744.

[9] Timoshenko, S.P., "On the corrections for shear of the differential equation for transverse vibrations of prismatic bars", Philosophical Magazine, Vol. 41, 1921, pp. 744--746.

[10] Novozhilov, V.V., Theory of elasticity, Pergamon Press, Oxford, 1961.

[11] Timoshenko, S.P., and Goodier, J.N., Theory of elasticity, McGraw-Hill, New York, 1970.

[12] Sokolnikoff, I.S., Mathematical Theory of Elasticity, McGrw-Hill, 1956.

[13] Cowper, G.R., “The Shear Coefficient in Timoshenko's Beam Theory", Journal of Applied Mechanics, Vol. 33, No. 2, 1966, pp. 335--340.

[14] Krishna Murty A.V., "On the Shear Deformation Theory for Dynamic Analysis of Beams”, Journal of Sound and Vibration, Vol. 101, No. 1, 1985, pp. 1--12.

[15] Pai, P.F., and Schulz, M.J., "Shear correction factors and an-energy consistent beam theory", International Journal of Solids and Structures, Vol. 36, 1999, pp. 1523--1540.

[16] Mechab, I., Tounsi, A., Benatta, M.A., and Adda Bedia, E.A., "Deformation of short composite beam using refined theories", Journal of Mathematical Analysis and Applications,Vol. 36, No. 6, 2008, pp. 468--479.

[17] Gruttmann, F., Sauer, R., and Wagner, W., "Shear Stresses in Prismatic Beams with Arbitrary Cross-Sections", International Journal for Numerical Methods in Engineering, Vol. 45, 1999, pp. 865--889.

[18] El, Fatmi R., and Ghazouani, N., "Higher order composite beam theory built on Saint-Venant $s^{\prime} s^{\prime}$ solution. Part-I: Theoretical developments", Composite Structures, Vol. 93, No. 2, 2011, pp. 557-566, Doi:10.1016/j. compstruct.2010.08.024.

[19] Krayterman, B.L., and Krayterman, A.B., "Generalized nonuniform torsion of beams and frames", Journal of Structural Engineering, Vol. 113, 1987, pp. 1772--1787.

[20] Ladéveze, P., Sanchez, P., and Simmonds, J., "Beamlike (Saint-Venant) solutions for fully anisotropic elastic tubes of arbitrary closed cross section", International Journal of Solids \& Structures, Vol. 41, No. 7, 2004,pp. 1925--1944.

[21] Ie囚an, D., “On Saint-Venant's problem”, Archive for Rational Mechanics and Analysis, Vol. 91, 1986, pp. 363-373.

[22] Lin, H.C., and Dong, S.B., "On the Almansi-Michell problems for an inhomogeneous, anisotropic cylinder", Journal of Mechanics, Vol. 22, No. 1, 2006, pp. 51--57.

[23] Berdichevsky, V.L., Armanios, E., and Badir, A., "Theory of anisotropic thin-walled closed-cross-section beams", Composites Engineering, Vol. 2, No. 5-7, 1992, pp.
411--432.

[24] Volovoi, V.V., Hodges, D.H., Berdichevsky, V.L., and Sutyrin, V.G. "Asymptotic theory for static behavior of elastic anisotropic I-beams", International Journal of Solid Structures, Vol. 36, No. 7, 1999,pp. 1017--1043.

[25] Volovoi, V.V., and Hodges, D.H., "Theory of anisotropic thin-walled beams", Journal of Applied Mechanics, Vol. 67, No. 3, 2000, pp. 453--459.

[26] Yu, W., Volovoi, V.V., Hodges, D.H., and Hong, X., "Validation of the variational asymptotic beam sectional analysis (VABS)", AIAA Journal, Vol. 40, No. 10, 2002, pp. 2105--2113.

[27] Schardt, R., "Eine Erweiterung der Technischen Biegetheorie zur Berechnung prismatischer Faltwerke”, Der Stahlbau, Vol. 35, 1966, pp. 161--171.

[28] Silvestre, N., "Generalised beam theory to analyse the buckling behaviour of circular cylindrical shells and tubes", Thin-Walled Structures, Vol. 45, No. 2, 2007, pp. 185--198.

[29] Nedelcu, M., "GBT formulation to analyse the behaviour of thin-walled members with variable crosssection", Thin-Walled Structures, Vol. 48, No. 8, 2010, pp. 629-638 .

[30] Washizu, K., Variational methods in elasticity and plasticity, Pergamon Press, Oxford, 1968.

[31] Kanok-Nukulchai, W., and Shik Shin, Y., "Versatile and improved higher-order beam elements", Journal of Structural Engineering, Vol. 110, 1984, pp. 2234--2249.

[32] Kapania, K., and Raciti, S., "Recent Advances in Analysis of Laminated Beams and Plates, Part I: Shear Effects and Buckling", AIAA Journal, Vol. 27, No. 7, 1989, pp. 923-935.

[33] Kapania, K., and Raciti, S., "Recent Advances in Analysis of Laminated Beams and Plates, Part II: Vibrations and Wave propagation", AIAA Journal, Vol. 27, No. 7, 1989, pp. 935--946.

[34] Librescu, L., and Song, O., "On the static aeroelastic tailoring of composite aircraft swept wings modelled as thinwalled beam structures", Composites Engineering, Vol. 2, 1992, pp. 497--512.

[35] Qin, Z., and Librescu, L., "On a shear-deformable theory of anisotropic thin-walled beams: further contribution and validations", Composite Structures, Vol. 56, 2002, pp. 345$-358$.

[36] Banerjee, J.R., Su, H., and Jayatunga, C., "A dynamic stiffness element for free vibration analysis of composite beams and its application to aircraft wings", Computers and Structures, Vol. 86, 2008, pp. 573--579.

[37] Carrera, E., Giunta, G., and Petrolo, M., Beam Structures: Classical and Advanced Theories, John Wiley \& Sons, Chicester, UK, 2011.

[38] Carrera, E., "Theories and finite elements for multilayered, anisotropic, composite plates and shells", 
Archives of Computational Methods in Engineering, Vol. 9, No. 2, 2002, pp. 87--140.

[39] Carrera E., "Theories and finite elements for multilayered plates and shells: a unified compact formulation with numerical assessment and benchmarking", Archives of Computational Methods in Engineering, Vol. 10, No. 3, 2003, pp. 216--296.

[40] Carrera, E., and Giunta, G., "Refined beam theories based on a unified formulation", International Journal of Applied Mechanics, Vol. 2, No., 1, 2010, 2(1): pp. 117--143

[41] Carrera, E., Giunta, G., Nali, P., and Petrolo, M., "Refined beam elements with arbitrary cross-section geometries", Computers and Structures, Vol. 88, No. 5-6, 2010, pp. 283-293, DOI: 10.1016/j.compstruc.2009.11.002.

[42] Carrera, E., Giunta, G., and Petrolo, M., "A Modern and Compact Way to Formulate Classical and Advanced Beam Theories", Developments and Applications in Computational Structures Technology, edited by Adam, J.M., Pallarés, F.J., Bru, R., and Romero, M.L., Saxe-Coburg Publications, Stirlingshire, UK, 2010, pp 75-112. doi:10.4203/csets.25.4

[43] Carrera, E., Petrolo, M., and Zappino, E., "Performance of CUF approach to analyze the structural behavior of slender bodies", Journal of Structural Engineering, Vol. 138, No. 2, 2012, pp. 285-297,Doi:10.1061/(ASCE)ST.1943541X.0000402.

[44] Carrera, E., Petrolo, M., and Nali, P., "Unified formulation applied to free vibrations finite element analysis of beams with arbitrary section", Shock and Vibrations, Vol. 17, 2010, pp. 1-18, DOI: 10.3233/SAV-2010-0528.

[45] Carrera, E., Petrolo, M., and Varello, A., "Advanced Beam Formulations for Free Vibration Analysis of Conventional and Joined Wings", Journal of Aerospace Engineering, Vol. 25, No. 2, 2012, pp. 282-293,Doi:10.1061/ (ASCE)AS.1943-5525.0000130.

[46] Petrolo, M., Zappino, E., and Carrera, E., "Unified higher-order formulation for the free vibration analysis of one-dimensional structures with compact and bridge-like cross-sections", Thin Walled Structures, Vol. 56, 2012, pp. 4961,Http://dx.doi.org/10.1016/j.tws.2012.03.011.

[47] Ibrahim, S.M., Carrera, E., Petrolo, M., and Zappino, E., "Buckling of composite thin walled beams by refined theory", Composite Structures, Vol. 94, No. 2, 2012, pp. 563-570.

[48] Ibrahim, S.M., Carrera, E., Petrolo, M., and Zappino, E., "Buckling of thin walled beams by refined theory", Journal ofZhejiang University Science A, 2012, In press, DOI: 10.1631/ jzus.A1100331.

[49] Carrera, E., and Petrolo, M., "Refined Beam Elements with only Displacement Variables and Plate/ Shell Capabilities", Meccanica, Vol. 47, No. 3, 2012, pp. 537556,DOI: 10.1007/s11012-011-9466-5.

[50] Carrera, E., and Petrolo, M., "Refined OneDimensional Formulations for Laminated Structure
Analysis", AIAA Journal, Vol. 50, No. 1, 2012, pp. 176-189, Doi: 10.2514/1.J051219.

[51] Carrera, E., Maiarù, M., and Petrolo, M., "ComponentWise Analysis of Laminated Anisotropic Composites", International Journal of Solids and Structures, Vol. 49, No. 13, 2012, pp. 1839-1851,Http://dx.doi.org/10.1016/j. ijsolstr.2012.03.025.

[52] Varello, A., Carrera, E., and Demasi, L., "Vortex Lattice Method Coupled with Advanced One-Dimensional Structural Models", ASDJournal, Vol. 2, No. 2, 2011, pp.53-78.

[53] Landahl, M.T., "Kernel Function for Nonplanar Oscillating Surfaces in a Subsonic Flow", AIAA Journal, Vol. 5, No. 5, 1967, pp. 1045--1046.

[54] Katz, J., and Plotkin, A., Low-Speed Aerodynamics: From Wing Theory to Panel Methods, McGraw-Hill, Inc., New York, 1991.

[55] Harder, R.L., and Desmarais, R.N., "Interpolation Using Surface Splines”, Journal of Aircraft, Vol. 9, No. 2, 1972, pp. 189--192.

[56] Demasi, L., and Livne, E., "Dynamic Aeroelasticity of Structurally Nonlinear Configurations Using Linear Modally Reduced Aerodynamic Generalized Forces", AIAA Journal, Vol. 47, No. 1, 2009, pp. 71-90

[57] Chen, P.C., "Damping Perturbation Method for Flutter Solution: The g-Method", AIAA Journal, Vol. 38, No. 9, 2000, pp. 1519--1524.

[58] Försching, H., Triebstein, H., and Wagener, J., "Pressure Measurements on an Harmonically Oscillating Swept Wing with Two Control Surfaces in Incompressible Flow", AGARD Conference Proceedings, Tønsberg, Norway, November 1970.

[59] Ueda, T., and Dowell, E.H., "A new solution method for lifting surfaces in subsonic flow", AIAA Journal, Vol. 20, No. 3, 1982, pp. 348--355.

[60] Rowe, W.S., and Petrarca, J.R., "Reduction of computer usage costs in predicting unsteady aerodynamic loadings caused by control surface motions", NASA, 1980, 145354-1. [61] Koo, K.N., "Aeroelastic Characteristics of Double-Swept Isotropic and Composite Wings", Journal of Aircraft, Vol. 38, No. 2, 2001, pp. 343--348.

[62] Kornecki, A., Dowell, E.H., and O'Brien, J., “On the aeroelastic instability of two-dimensional panels in uniform incompressible flow", Journal of Sound and Vibration, Vol. 47, No. 2, 1976, pp. 163-178.

[63] Attaran, A., Majid, D.L., Basri, S., Mohd Rafie, A.S., and Abdullah, E.J., "Structural optimization of an aeroelastically tailored composite flat plate made of woven fiberglass/ epoxy", Aerospace Science and Technology, Vol. 15, 2011; pp. 393-401. 